\title{
Evaluation of Clinical Process by the National Case-mix Database in Japan
}

\section{-An Example of Evaluation of the Compliance with Clinical Practice Guidelines for Acute Cholangitis-}

\author{
Tatsuhiko Kubo ${ }^{1)}$, Atsuhiko Murata' ${ }^{1)}$, Yoshihisa Fujino ${ }^{1)}$, Shinya Matsuda ${ }^{1)}$ \\ ${ }^{1)}$ Department of Preventive Medicine and Community Health, School of Medicine, University of Occupational \\ and Environmental Health, Japan
}

\begin{abstract}
It is an important mission for health policy makers to assure the quality care for public. This requires some evaluation methods. The compliance level of clinical practice guidelines (CPGs) will be one of the possible tools to evaluate the quality of clinical process. However, it is not an easy task to systematically monitor the compliance level. As the Japanese DPC database gathers very detailed process information, it is possible to evaluate the CPGs compliance level. In this article, the authors would like show the usability of DPC database for process evaluation based on our previous literatures.
\end{abstract}

Key words: DPC, clinical practice guideline, quality of care, evaluation

\section{Introduction}

It is an important mission for health policy makers to assure the quality care for public. Under the current difficulty in economic situation, it is also important for policy makers to control the health expenditures. As a poor functional differentiation is considered as one of the possible reasons for inefficient resource use in the Japanese health system, the Ministry of Health, Labour and Welfare (MHLW) has tried to re-organize the health system by the establishment of Regional Health Plan since 1984. The centralization and functional differentiation are cores of such a policy. Behind this policy, there is a hypothesis of volumeoutcome relationship.

The volume-outcome relationship has been intensively investigated for the past decades. According to

\footnotetext{
Received: September 16, 2011

Accepted: February 1, 2012

Correspondence: T. Kubo, Department of Preventive Medicine and Community Health, School of Medicine, University of Occupational and Environmental Health, 1-1 Iseigaoka, Yahatanishi-ku, Kitakyushu, Fukuoka 807-8555, Japan e-mail: kubo@med.uoeh-u.ac.jp
}

the systematic review by Halm et al, high volume is associated with better outcomes across a wide range of procedures and conditions, but the magnitude of the association varies greatly ${ }^{1}$. They have suggested that differences in case mix and processes of care between high- and low-volume providers might explain part of the observed relationship between volume and outcome. Thus it is necessary to investigate the relationship between volume and quality of clinical processes. The compliance level of clinical practice guidelines (CPGs) will be one of the possible tools to evaluate the quality of clinical process.

In fact, there have been many previous studies into the relationship between patient outcome and compliance with $\mathrm{CPGs}^{2-5)}$. For example, Quaglini et $a l$. reported that guideline compliance was a significant independent indicator of medical cost and length of stay (LOS) in patients with stroke. Other studies have also reported that higher compliance with CPGs related to the better clinical outcomes, such as lower in-hospital mortality and shorter LOS.

However, to our knowledge, there have been little literatures that focused into the relationship among hospital volume, compliance with CPGs and outcome. 
Table 1 Contents of Form1 Minimum Data Set

\begin{tabular}{|c|c|c|}
\hline Hospital ID & Complication 1 & Japan Coma Scale at admission \\
\hline Division ID & Complication 2 & Japan Coma Scale at discharge \\
\hline Summary ID & Complication 3 & Recurrent cancer \\
\hline Record ID & Complication 4 & $\mathrm{UICC}(\mathrm{T})$ \\
\hline Sex & Surgical procedure code 1 & $\mathrm{UICC}(\mathrm{N})$ \\
\hline Birth Date & Operation site & UICC (M) \\
\hline ZIP code (Patient) & Type of anesthesia & Cancer Stage \\
\hline Purpose & Date & modified Rankin Scale at admission \\
\hline Clinical trial & Surgical procedure code 2 & modified Rankin Scale at discharge \\
\hline Admission Date & Operation site & Hugh-Jones classification \\
\hline Discharge Date & Type of anesthesia & NYHA classification \\
\hline Referral to other wards & Date & CCS classification \\
\hline Admission Pass & Surgical procedure code 3 & Killips classification \\
\hline Referral from other facilities & Operation site & Severity classification of pneumonia \\
\hline $\begin{array}{l}\text { Admission from out-patient service } \\
\text { of the hospital }\end{array}$ & Type of anesthesia & Child-Pugh classification \\
\hline Planned admission & Date & Severity classification of acute pancreatitis \\
\hline Ambulance service use & Surgical procedure code 4 & Burn Index \\
\hline Discharge Pass & Operation site & Other classification for severity, if any \\
\hline Outcome at discharge & Type of anesthesia & Gestational weeks at admission \\
\hline Death within $24 \mathrm{~h}$ from admission & Date & $\begin{array}{l}\text { Admission status under the Mental health } \\
\text { and welfare low (MHWL) }\end{array}$ \\
\hline Latest admission (Date) & Surgical procedure code 5 & Isoration duration by MHWL (days) \\
\hline Latest admission by same Dx & Operation site & Physical restraint duration by MHWL (days) \\
\hline Principal Diagnosis & Type of anesthesia & GAF score at admission \\
\hline Diagnosis for admission & Date & Additional code for diagnosis, if any \\
\hline Diagnosis of the most resource used & Current pregnancy status & Chemotherapy \\
\hline Diagnosis of the second most resource used & Birth weight & \\
\hline Co-Morbidity 1 & Gestational weeks at birth & \\
\hline Co-Morbidity 2 & Height & \\
\hline Co-Morbidity 3 & Weight & \\
\hline Co-Morbidity 4 & Smoking index & \\
\hline
\end{tabular}

In Japan, an original case-mix system so-called DPC (Diagnosis Procedure Combination) has been developed and used for hospital evaluation since 2005. This dataset contains a detailed procedure information. Using this dataset we have evaluated the relationship between hospital volume and compliance with CPGs for acute cholangitis ${ }^{6}$. The results are very interesting in showing that hospital volume was significantly correlated with compliance with CPGs and that compliance level was significantly correlated with in-hospital mortality ${ }^{6}$. In this article, the authors would like to explain the usability of Japanese casemix database (DPC database) for health economics and health politics studies by referring our previous literatures.

\section{About DPC Database}

As the principle of Japanese health insurance scheme has long been the fee-for-service (FFS) based payment, the health information companies have devel- oped the computer system corresponding to the FFS payment. Using the installed tariff table data, the computer produces a receipt (claim data) of each patient for reimbursement. Health institutions send this claim data to the payers' organization in order to receive reimbursement. In this computer system, all procedures, drugs and devices for reimbursement are registered for each patient by daily basis. There is a MHLW standard code for each of all procedures, drugs and devices.

The DPC database gathers these detailed electronic data as Form 1 Minimum Dataset (Form 1), Efile (cost data) and F-file (detailed procedure data). Table 1 shows the format of Form 1. Form 1 contains the following patient information; data ID number, birth date, sex, principal diagnosis (ICD-10), co-morbidity and complication (ICD-10), surgical intervention (Japanese payment code), other major procedures (Japanese payment code), emergency case or not, and outcome. The registration of above mentioned information is obligatory. Furthermore, there are faculta- 
Table 2 Contents of E-file and F-file

\section{E file}

Data matching

\begin{tabular}{|c|c|c|}
\hline \multirow{4}{*}{$\stackrel{\leftrightarrow \longmapsto}{\longleftrightarrow}$} & $\mathrm{F}-1$ & Hospital ID \\
\hline & $\mathrm{F}-2$ & Data ID number \\
\hline & $\mathrm{F}-3$ & Discharge Date \\
\hline & $\mathrm{F}-4$ & Admission Date \\
\hline \multirow{15}{*}{$\longleftrightarrow$} & $\mathrm{F}-5$ & Data category \\
\hline & $\mathrm{F}-6$ & Data serial number \\
\hline & $\mathrm{F}-7$ & Procedure serial number for the same $E-6$ \\
\hline & $\mathrm{F}-8$ & Master code of hospital tariff table \\
\hline & $\mathrm{F}-9$ & Code for claim data processing system \\
\hline & $\mathrm{F}-10$ & Code of tariff table \\
\hline & $\mathrm{F}-11$ & Name of procedure \\
\hline & $\mathrm{F}-12$ & Volume of procedure \\
\hline & $\mathrm{F}-13$ & Unit of prpcedure \\
\hline & $\mathrm{F}-14$ & Tariff point of procedure \\
\hline & $\mathrm{F}-15$ & Tariff point for drugs \\
\hline & $\mathrm{F}-16$ & Tariff point for devices \\
\hline & $\mathrm{F}-17$ & Code for point or yen \\
\hline & $\mathrm{F}-18$ & Points for reimbursement \\
\hline & $\mathrm{F}-19$ & Flag for Fee for service payment \\
\hline
\end{tabular}

\begin{tabular}{|c|c|}
\hline $\mathrm{E}-1$ & Hospital ID \\
\hline $\mathrm{E}-2$ & Data ID number \\
\hline $\mathrm{E}-3$ & Discharge Date \\
\hline $\mathrm{E}-4$ & Admission Date \\
\hline $\mathrm{E}-5$ & Data category \\
\hline E-6 & Data serial number \\
\hline E-7 & Master code of hospital tariff table \\
\hline $\mathrm{E}-8$ & Code for claim data processing system \\
\hline E-9 & Code of tariff table \\
\hline $\mathrm{E}-10$ & Name of procedure \\
\hline $\mathrm{E}-11$ & Tariff point of procedure \\
\hline $\mathrm{E}-12$ & Tariff point for drugs \\
\hline$E-13$ & Tariff point for devices \\
\hline $\mathrm{E}-14$ & Code for point or yen \\
\hline $\mathrm{E}-15$ & Frequency of procedures \\
\hline $\mathrm{E}-16$ & Code of insurer \\
\hline $\mathrm{E}-17$ & Code of claim type \\
\hline $\mathrm{E}-18$ & Undertaking Date \\
\hline $\mathrm{E}-19$ & Claim classification \\
\hline $\mathrm{E}-20$ & Clinical division's code \\
\hline $\mathrm{E}-21$ & Doctor's code \\
\hline $\mathrm{E}-22$ & Ward's code \\
\hline $\mathrm{E}-23$ & Classification of ward \\
\hline $\mathrm{E}-24$ & In-patient or Out-patient \\
\hline $\mathrm{E}-25$ & Tyoe of facility \\
\hline
\end{tabular}

\section{F file}

For each procedure, drug and device, F-file is created. These F-files data are grouped into the corresponding E-file (that represents a procedure group).

tive information such as ADL score (Barthel index), severity score such as NYHA and Killips score, cancer staging, UICC code and other clinical indicators.

Table 2 shows the formats of E-fail and F-file. For each procedure, drug and device, F-file is created. These F-file data are grouped into the corresponding E-file that represents a procedure group. E-file and Ffile is combined by Data ID number, admission date, discharge date, data category and Data serial number (Table 3). As shown in Table 4, detail of antibiotics use can be described, i.e., date and duration of use, volume, combination of treatment. In this way, we can analyze the process of each case in comparison with CPGs.

\section{* Empirical Results from DPC National Database on Evaluation of the Relationship between Hospital Volume and Compliance with CPGs for Acute Cholangitis}

In order to clarify the usefulness of DPC database for health service researches, we have conducted a study project that tried to evaluate the relationship between hospital volume and compliance with CPGs. We selected CPGs for acute cholangitis ${ }^{6}$. These CPGs were posted in the Journal of Heapto-Biliary-Pancreatic Surgery in 2007 ${ }^{7-12)}$. These CPGs are the world's first international guidelines for acute cholangitis and have been awaiting evaluation. The outline of study design and results are as following (more detailed information is available in our previous literature ${ }^{6}$. Table 5 shows the selected recommendations used for our analyses.

This study was an observational one based on 60,842 acute cholangitis cases (July to December 2008) from 829 acute care hospitals in Japan. Hospital volume was categorized into the three groups; lowvolume hospitals (LVHs: number of cases $=20,869$, number of hospitals $=499$ ) that had less than 80 cases, medium-volume hospitals (MVHs: number of cases $=18,387$, number of hospitals $=188$ ) that had 80 to 120 cases and high-volume hospitals (HVHs: number of cases $=21,586$, number of hospitals $=142$ ) that have more than 120 cases. As shown in Figure 1, patients were categorized into the three grades (Grade I, II, III) 
Table 3 Structure of E-file and F-file (extracted)

\section{E-file}

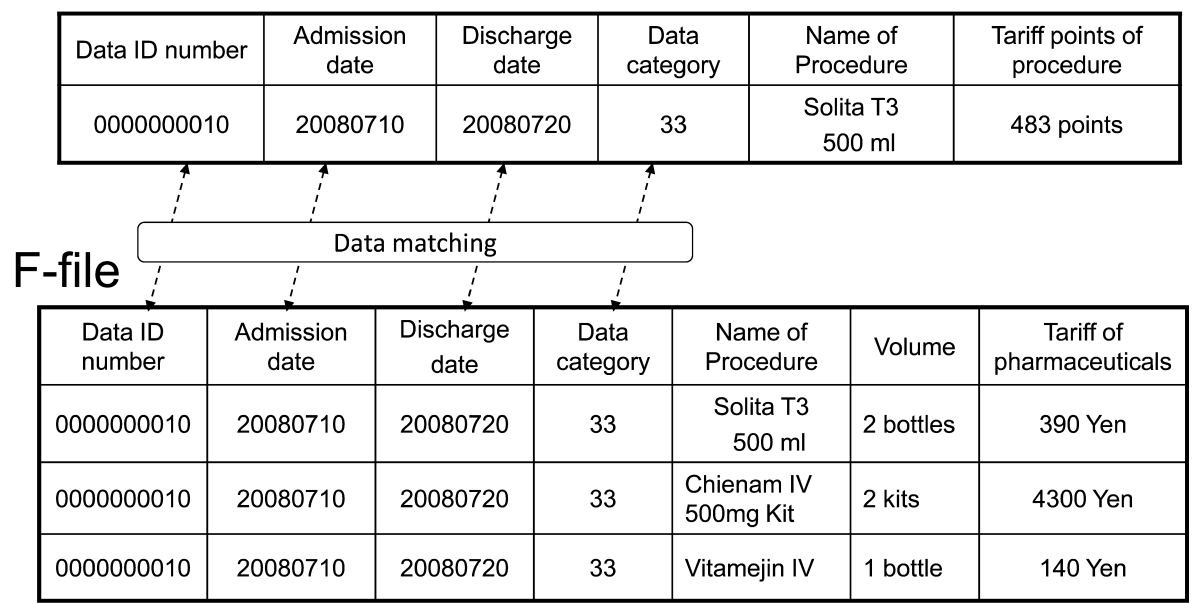

Data ID number is the number of each discharge case. This ID is the same as Form 1.

Table 4 An example of process analysis based on E-file and F-file

\section{F file}

\begin{tabular}{|c|c|}
\hline $\mathrm{F}-1$ & Hospital ID \\
\hline $\mathrm{F}-2$ & Data ID number \\
\hline $\mathrm{F}-3$ & Discharge Date \\
\hline $\mathrm{F}-4$ & Admission Date \\
\hline $\mathrm{F}-5$ & Data category \\
\hline $\mathrm{F}-6$ & Data serial number \\
\hline $\mathrm{F}-7$ & Procedure serial number for the same $E-6$ \\
\hline $\mathrm{F}-8$ & Master code of hospital tariff table \\
\hline $\mathrm{F}-9$ & Code for claim data processing system \\
\hline $\mathrm{F}-10$ & Code of tariff table \\
\hline $\mathrm{F}-11$ & Name of procedure \\
\hline $\mathrm{F}-12$ & Volume of procedure \\
\hline $\mathrm{F}-13$ & Unit of prpcedure \\
\hline $\mathrm{F}-14$ & Tariff point of procedure \\
\hline $\mathrm{F}-15$ & Tariff point for drugs \\
\hline $\mathrm{F}-16$ & Tariff point for devices \\
\hline $\mathrm{F}-17$ & Code for point or yen \\
\hline $\mathrm{F}-18$ & Points for reimbursement \\
\hline $\mathrm{F}-19$ & Flag for Fee for service payment \\
\hline \multicolumn{2}{|r|}{ E file } \\
\hline$E-13$ & Tariff point for devices \\
\hline$E-14$ & Code for point or yen \\
\hline $\mathrm{E}-15$ & Frequency of procedures \\
\hline$E-16$ & Code of insurer \\
\hline$E-17$ & Code of claim type \\
\hline E-18 & Undertaking Date \\
\hline$E-19$ & Claim classification \\
\hline
\end{tabular}

\begin{tabular}{|c|c|c|c|}
\hline$\downarrow$ & & \multicolumn{2}{|c|}{$\begin{array}{c}\text { Pharmaceutical } \\
\text { Master table }\end{array}$} \\
\hline$\nabla$ & $\forall$ & $\nabla$ & $\nabla$ \\
\hline Data ID number & antibiotics & Days_after_admission & Volume (mg) \\
\hline 1234567890 & $\mathrm{CPZ} / \mathrm{SBT}$ & 1 & 1,000 \\
\hline 1234567890 & $\mathrm{CPZ} / \mathrm{SBT}$ & 2 & 2,000 \\
\hline 1234567890 & $\mathrm{CPZ} / \mathrm{SBT}$ & 3 & 2,000 \\
\hline 1234567890 & $\mathrm{CPZ} / \mathrm{SBT}$ & 4 & 2,000 \\
\hline 1234567890 & $\mathrm{CPZ} / \mathrm{SBT}$ & 5 & 2,000 \\
\hline 1234567890 & $\mathrm{CPZ} / \mathrm{SBT}$ & 6 & 2,000 \\
\hline 1234567890 & $\mathrm{CPZ} / \mathrm{SBT}$ & 7 & 2,000 \\
\hline 1234567890 & $\mathrm{CPZ} / \mathrm{SBT}$ & 8 & 2,000 \\
\hline 2345678901 & $\mathrm{CPZ} / \mathrm{SBT}$ & 3 & 2,000 \\
\hline 2345678901 & $\mathrm{CPZ} / \mathrm{SBT}$ & 4 & 2,000 \\
\hline 3456789012 & $\mathrm{CPZ} / \mathrm{SBT}$ & 2 & 1,000 \\
\hline 3456789012 & $\mathrm{CPZ} / \mathrm{SBT}$ & 3 & 2,000 \\
\hline 4567890123 & $\mathrm{CPZ} / \mathrm{SBT}$ & 2 & 1,000 \\
\hline 4567890123 & $\mathrm{CPZ} / \mathrm{SBT}$ & 3 & 2,000 \\
\hline 5678901234 & $\mathrm{CPZ} / \mathrm{SBT}$ & 5 & 1,000 \\
\hline 5678901234 & $\mathrm{CPZ} / \mathrm{SBT}$ & 6 & 2,000 \\
\hline 6789012345 & CZOP & 1 & 1,000 \\
\hline 6789012345 & CZOP & 2 & 1,000 \\
\hline 6789012345 & IPM/CS & 2 & 500 \\
\hline 6789012345 & IPM/CS & 3 & 1,000 \\
\hline 6789012345 & IPM/CS & 4 & 1,000 \\
\hline 6789012345 & $\mathrm{CPZ} / \mathrm{SBT}$ & 5 & 1,000 \\
\hline 6789012345 & IPM/CS & 5 & 1,000 \\
\hline 6789012345 & $\mathrm{CPZ} / \mathrm{SBT}$ & 6 & 2,000 \\
\hline 6789012345 & IPM/CS & 6 & 1,000 \\
\hline 6789012345 & IPM/CS & 7 & 1,000 \\
\hline 6789012345 & IPM/CS & 8 & 1,000 \\
\hline 6789012345 & IPM/CS & 9 & 1,000 \\
\hline 6789012345 & IPM/CS & 10 & 1,000 \\
\hline 6789012345 & IPM/CS & 11 & 1,000 \\
\hline 6789012345 & IPM/CS & 12 & 1,000 \\
\hline
\end{tabular}

From E-file and F-file, the database for analysis is created in this way. 


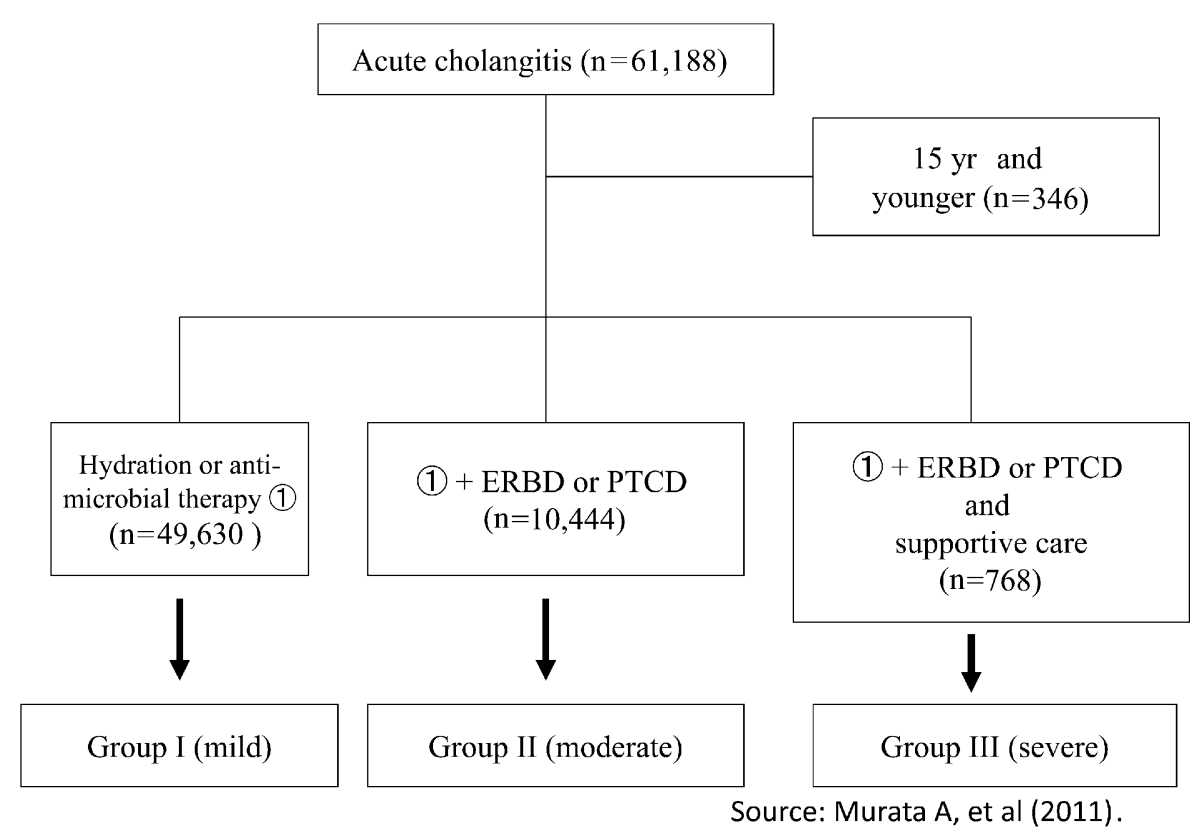

Figure 1 Severity classification based on DPC data

Table 5 Selected recommendations in the clinical practice guidelines (CPGs) for acute cholangitis

Recommendation A

(1) Antimicrobial agents should be administered intravenously to patients diagnosed as having acute cholangitis.

(2) Antimicrobial drugs should be selected according to the severity of acute cholangitis.

(3) Biliary penetration should be considered in the selection of antimicrobial agents in acute cholangitis.

(4) For patients with mild (grade I) acute cholangitis, the duration of antimicrobial therapy could be shorter (2 or 3 days).

(5) For patients with moderate (grade II) or severe (grade III) acute cholangitis, antimicrobial agents should be administered for a minimum duration of 5-7 days.

(6) Endoscopic biliary drainage should be selected for biliary decompression.

(7) Patients with acute cholangitis, especially those with severe (grade III) disease, should have immediate biliary drainage.

Recommendation B

(8) Bile cultures should be performed at all available opportunities.

(9) Blood cultures should be performed at all available opportunities.

(10) Cholecystectomy is indicated after the resolution of acute cholangitis.

according to the guidelines definition; Grade I is mild acute cholangitis that responds to initial medical treatment such as hydration or antimicrobial therapy, grade II is moderate acute cholangitis that does not respond to initial medical treatment and require biliary drainage, and grade III is severe case that requires initial treatment, biliary drainage and organ support.

The compliance rates of the recommendations were calculated for each patient as follows ( 0 to $100 \%$ );

For grade I; 6 recommendations in Table 5 -item (1) to (4) and (9), (10),

For grade II and III; 9 recommendations - item (1) to (3) and (5) to (10)
We have conducted a multiple logistic regression in order to evaluate the relationship between compliance with CPGs and in-hospital mortality as a clinical outcome, and a liner regression analysis for the investigation on the relationship between hospital volume and CPGs score. The results have showed that hospital volume was the most significant factors among all variables in predicting CPGs compliance (standardized coefficient for HVHs is $0.689, p<0.0001$ ), after adjusting for potential confounding effects of demographic and clinical variables. 


\section{Conclusion}

As our previous research results have showed, the DPC database is a useful tool for process evaluation. To our knowledge, there is no other administrative database that systematically gathers the detailed process information at the same level as DPC database. It is no doubt that the Japanese DPC database is a very powerful dataset for health service researches. But it has some limitations. For example, the CPGs of acute cholangitis recommend the estimation of serum creatinine clearance and the management of drug dosage in accordance with presenting renal function ${ }^{9}$, but laboratory data and imaging findings were not available in the DPC database ${ }^{13,14)}$. Furthermore, as the DPC database covers only in-patient period, it is not possible to know preand post-hospitalization conditions. In order to overcome these limitations, we plan to establish a research framework that uses DPC database as a tool for case detection and organizes an additional research of the detected cases for more detailed clinical information.

Clinical governance has been recently introduced as a systematic approach to maintaining and improving the quality of patient care in the health care system $^{15)}$. There is no doubt that the monitoring of CPGs compliance is useful evidence for maintaining and improving quality of medical care as a measure of quality improvement ${ }^{2-5}$. As our previous study demonstrated $^{6}$, some hospitals had poor compliance with CPGs. The use of the Japanese DPC database enabled the identification of the hospitals with insufficient adherence to CPGs to occur.

\section{References}

1) Halm EA, Lee $C$, Chassin MR: MPH is volume related to outcome in health care? A systematic review and methodologic critique of the literature. Annals of Internal Medicine 137, 511-520 (2002).

2) Quaglini S, Cavallini A, Gerzeli S, Micieli G: Economic benefit from clinical practice guideline compliance in stroke patient management. Health Policy 69, 305-315 (2004).

3) Ellrodt AG, Conner L, Riedinger M, Weingarten S: Measuring and improving physician compliance with clinical practice guidelines. A controlled interventional trial. Ann Intern Med 122, 277-282 (1995).

4) Micieli G, Cavallini A, Quaglini S: Guideline compliance improves stroke outcome: a preliminary study in 4 districts in the Italian region of Lombardia. Stroke 33, 1341-1347 (2002).

5) Gulati M, Patel S, Jaffe AS, Joseph AJ, Calvin JE Jr.: Impact of contemporary guideline compliance on risk stratification models for acute coronary syndromes in The Registry of Acute Coronary Syndromes. Am J Cardiol 94, 873-878 (2004).

6) Murata A, Matsuda S, Kuwabara K, Fujino Y, Kubo T, Fujimori K, et al.: An observational study using a national administrative database to determine the impact of hospital volume on compliance with clinical practice guidelines. Medical Care 49, 313-320 (2011).

7) Takada $T$, Kawarada $Y$, Nimura $Y$, Yoshida $M$, Mayumi T, Sekimoto M, et al.: Background: Tokyo Guidelines for the management of acute cholangitis and cholecystitis. J Hepatobiliary Pancreat Surg 14, 1-10 (2007).

8) Kimura $Y$, Takada $T$, Kawarada $Y$, Nimura $Y$, Hirata K, Sekimoto M, et al.: Definitions, pathophysiology, and epidemiology of acute cholangitis and cholecystitis: Tokyo Guidelines. J Hepatobiliary Pancreat Surg 14, 15-26 (2007).

9) Tanaka A, Takada T, Kawarada Y, Nimura Y, Yoshida M, Miura F, et al.: Antimicrobial therapy for acute cholangitis: Tokyo Guidelines. J Hepatobiliary Pancreat Surg 14, 59-67 (2007).

10) Nagino $M$, Takada $T$, Kawarada $Y$, Nimura $Y$, Yamashita $\mathrm{Y}$, Tsuyuguchi T, et al.: Methods and timing of biliary drainage for acute cholangitis: Tokyo Guidelines. J Hepatobiliary Pancreat Surg 14, 68-77 (2007).

11) Yasuda $H$, Takada $T$, Kawarada $Y$, Nimura $Y$, Hirata K, Kimura Y, et al.: Unusual cases of acute cholecystitis and cholangitis: Tokyo Guidelines. $\mathrm{J}$ Hepatobiliary Pancreat Surg 14, 98-113 (2007).

12) Mayumi $T$, Takada $T$, Kawarada $Y$, Nimura $Y$, Yoshida M, Sekimoto M, et al.: Results of the Tokyo Consensus Meeting Tokyo Guidelines. J Hepatobiliary Pancreat Surg 14, 114-121 (2007).

13) Matsuda S: Casemix as a tool for transparency of medical services. Jpn J Soc Secur Policy 6, 43-53 (2008).

14) Matsuda $S$, Ishikawa KB, Kuwabara $K$, Fujimori $K$, Fushimi K, Hashimoto H, et al.: Development and use of the Japanese case-mix system. Eurohealth 14, 25-30 (2008).

15) Salter B: Governing UK medical performance: a struggle for policy dominance. Health Policy 82 , 263-275 (2007). 